\title{
Driving mechanism of keyhole evolution during multi-pulse drilling with a millisecond laser
}

\author{
Yue Zhang ${ }^{1,3}$, Gang $\mathrm{Yu}^{1,2,3^{*}}$, XiuLi He${ }^{1,3^{*}}$, ShaoXia $\mathrm{Li}^{1,3}$, and WeiJian Ning ${ }^{1}$ \\ ${ }^{1}$ Key Laboratory of Mechanics in Advanced Manufacturing, Institute of Mechanics, Chinese Academy of Sciences, Beijing 100190, China; \\ ${ }^{2}$ Center of Materials Science and Optoelectronics Engineering, University of Chinese Academy of Sciences, Beijing 100049, China; \\ ${ }^{3}$ School of Engineering Science, University of Chinese Academy of Sciences, Beijing 100049, China
}

Received March 11, 2019; accepted April 25, 2019; published online June 25, 2019

\begin{abstract}
To better understand the physical processes of multi-pulse laser drilling, this study investigates the keyhole evolution and its driving mechanism in a time-resolved observation system. The evolution characteristics suggested a two-phase process of rapid penetration followed by moderate penetration. As revealed in the ejection and vaporization behavior, the keyhole evolution was dominated by ejection and vaporization during the rapid and moderate penetration stages, respectively. In a single laser-pulsed drilling experiment, the driving mechanism itself was found to be affected by the dimensionless laser power density. The effect of dimensionless laser power density on depth increment was then discussed by comparing the experimental observations with numerical simulation results. The results further confirmed the driving mechanism of the keyhole evolution. The results in this paper are useful for understanding the driving mechanism of the keyhole evolution during multi-pulse laser drilling.
\end{abstract}

multi-pulse laser drilling, melt ejection, vaporization, keyhole evolution, driving mechanism

PACS number(s): 42.62.Cf, 68.18.Jk, 68.03.-g, 79.20.Ds

Citation: Y. Zhang, G. Yu, X. L. He, S. X. Li, and W. J. Ning, Driving mechanism of keyhole evolution during multi-pulse drilling with a millisecond laser, Sci. China-Phys. Mech. Astron. 62, 104711 (2019), https://doi.org/10.1007/s11433-019-9418-4

\section{Introduction}

Owing to its distinct advantages such as high flexibility, high machining rate, high accuracy, and repeatability, laser drilling is widely used in the mechanical, aerospace, electrical, and automotive engineering industries [1,2]. The laser-material interaction is a basic physical process in laser drilling. During the laser drilling, a fixed amount of energy is compressed into a short pulse duration, delivering a high peak power [3]. For ultra-short pulse lasers, such as femtosecond (fs) pulse lasers, the peak power is extremely high $\left(\geq 10^{13} \mathrm{~W} / \mathrm{cm}^{2}\right)$ and the irradiated material passes through a

\footnotetext{
*Corresponding authors (Gang Yu, email: gyu@imech.ac.cn; XiuLi He, email: xlhe@
} imech.ac.cn) nearly direct solid-vapor transition [4]. However, the material-removal rate [5] $\left(0.054 \mathrm{~mm}^{3} / \mathrm{min}\right.$ for a Clark-CPA 2010 femtosecond laser [6]) is much lower than that of long-pulse laser irradiation such as millisecond (ms) pulses $\left(27.4 \mathrm{~mm}^{3} / \mathrm{min}\right.$ for a Nd:YAG millisecond laser [7]). Under a millisecond pulse laser, the material goes through a solidliquid-vapor transition [4], and the molten material is ejected by the vaporization-induced recoil pressure [8,9], generating an unstable keyhole [10]. The keyhole instability is affected by multiple physical processes, such as the melt flow, vaporization at the keyhole front, homogeneous boiling, multiple internal reflections, and the melt droplet ejection [11-16].

In single-pulse laser drilling, the keyhole evolves after the initial melt ejection and mild melting. The hole expands, 
followed by backflow and recasting [10]. The whole process is affected by the bulging effect [15], shock waves, plasma dynamics [17,18], wave features at the keyhole front [19], and especially by the ejection and vaporization characteristics [20]. As the keyhole evolves, its front rapidly penetrates into greater depths as mass is lost by melt ejection and vaporization [10]. This material removal can be considered as the main driving mechanism of the keyhole evolution. $\mathrm{Ng}$ et al. [21] analyzed the process of material removal, and indicated that the hole forms mainly in the liquid-melt ejection phase rather than vaporization. Consequently, melt ejection is normally considered as the dominant driving mechanism during single-pulse drilling with millisecond pulse lasers.

A hole with a high aspect ratio is commonly formed by multi-pulse drilling. The aspect ratio of a multi-pulsed drilled hole can reach 1:20 [22]. However, multi-pulse drilling has more complex processing parameters than single-pulse drilling, and the ejection and vaporization behaviors are affected by the pulse number, repetition rate, and other factors [5]. The melt ejection may continue after the end of the pulse [7], leading to the melt shadowing effect [23] which greatly influences the keyhole evolution in subsequent pulses. Consequently, the characteristics of the keyhole evolution in multi-pulse laser drilling differ from those of single-pulse laser drilling. Tu et al. [3] investigated the physical processes during multi-pulse laser drilling, and indicated that the melt ejection becomes less effective beyond a certain position of the hole depth. Similar conclusions were reached after observing the ejection with high-speed photography [24,25]. However, as the evolving internal characteristics of the keyhole during multi-pulse drilling have not been observed, the driving mechanism of this keyhole evolution remains incompletely understood; particularly, at which point the melt ejection becomes less effective is unknown.

In this paper, the keyhole evolution was observed in a time-resolved observation system. The ejection and vaporization behaviors during the keyhole evolution were also observed. Finally, the driving mechanism of the keyhole evolution in multi-pulse drilling was investigated by comparing the experimental observations with numerical simulation results.

\section{Experiments}

The keyhole evolution during multi-pulse laser drilling was observed by a time-resolved observation system, as shown in Figure 1. The Gaussian laser pulses generated by a Nd:YAG pulsed laser system were transmitted by a $45^{\circ}$ reflecting mirror, and then focused on the top surface of the sample by a focal lens. Underneath the focal lens, the sample was fixed on a 3-axis CNC table by a clamp and irradiated by 10 laser pulses with a defocus distance of 0 , a wavelength of $1064 \mathrm{~nm}$, a pulse width of $0.6 \mathrm{~ms}$, a repetition rate of $100 \mathrm{~Hz}$, a laser power of $1600 \mathrm{~W}$, and a focal spot diameter of $0.22 \mathrm{~mm}$. For recording the whole keyhole evolution, the resolution, recording rate, and shutter speed of the highspeed camera were set to $192 \times 484$ pixels, $10000 \mathrm{f} / \mathrm{s}$ and $1 / 10000 \mathrm{~s}$, respectively.

For observing the keyhole evolution and the ejection and vaporization behavior over the hole entrance, the sample (a $0.2 \mathrm{~mm}$-thick 304 stainless steel sheet) was sandwiched between two clamps as seen in Figure 1(b). To weaken the extremely bright laser-induced light (Figure 1(c)) and filter out the refraction, a filter unit was placed between the sample and the high-speed camera. Also owing to the brightness of the laser light [26], it was difficult to judge whether the highlighted region inside the keyhole was caused by vapor or the laser-induced light. Hence, to observe the behavior of ejection and vaporization inside the keyhole, a $0.5 \mathrm{~mm}$ thick metal sheet was used and the laser spot was centered on the edge of the top surface (Figure 1(a)). Under this condition, the ejection and vapor inside the keyhole could be recorded.

\section{Results and discussion}

\subsection{Observation and characterization of the keyhole evolution}

The keyhole evolved during the on-time of the laser in the laser drilling process, and almost stopped growing when the laser was off. As the repetition rate of the pulsed laser was $100 \mathrm{~Hz}$, the interval between two adjacent pulses was $10 \mathrm{~ms}$. Meanwhile, the interval between two images recorded by the high-speed camera (operating at $10000 \mathrm{f} / \mathrm{s}$ ) was $0.1 \mathrm{~ms}$. Hence, 100 images were recorded during each laser pulse. The first six images recorded the keyhole evolution, and the remaining images captured the near-stationary keyhole.

When the laser was on, the keyhole evolution was recorded by the high-speed camera with the filter unit as shown in Figure 2. The filter unit comprised a band-pass filter admitting wavelengths from 400 to $600 \mathrm{~nm}$ and a neutral filter with 3\% transmittance, or the neutral filter only (omitting the band-pass filter). The keyhole observations through the neutral filter only and through the band-pass and neutral filter are shown in Figure 2(a) and (b), respectively. Because the light scattered from the laser irradiation was excessively strong, the camera was saturated when only the neutral filter was used, so the keyhole was unobservable. Hence, when the laser was on, both the band-pass and neutral filter were required.

However, when the laser was off, the temperature fell and the light weakened. In this situation, the images were clear when observed through the neutral filter alone (Figure 2(c)), but blurred when observed through both the band-pass and 


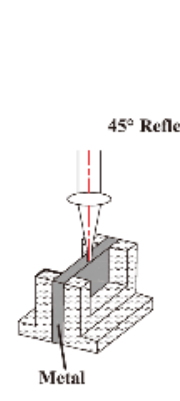

(a)

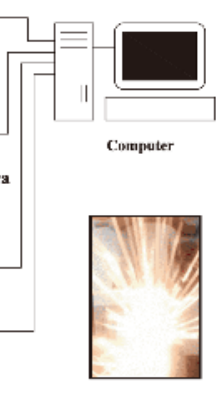

(c)
Figure 1 (Color online) Schematic of the time-resolved observation system. (a) Metal sheet; (b) "sandwich" structure; (c) laser induced light.
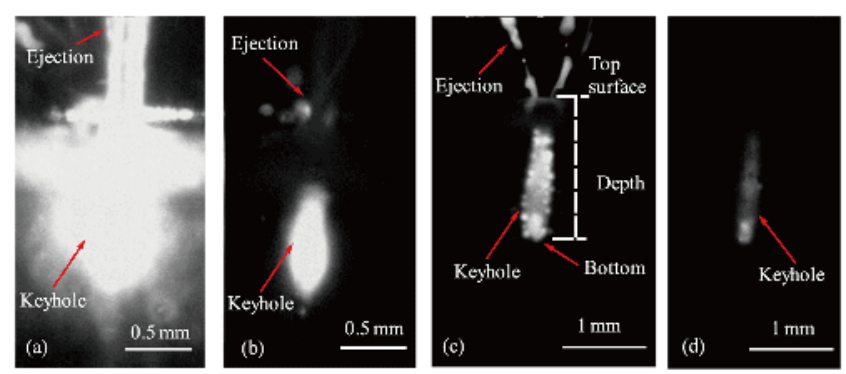

Figure 2 (Color online) Observations of keyhole through (a) the neutral filter and (b) both the band-pass and neutral filters during the on-time of the laser, and through (c) the neutral filter and (d) both the band-pass and neutral filters during the off-time of the laser.

neutral filters (Figure 2(d)). Hence, when the laser was off, the neutral filter alone was preferred for enhancing the image clarity.

Every multi-pulse laser drilling experiment was performed as described above, under the same conditions except for the filter unit. The keyhole evolution process during the on and off times of the laser could be recorded separately. Comparing Figure 2(b) and (c), the image acquired through both filters was much noisier during the on-time than during the off-time of the laser, and the image edge in Figure 2(c) is clearly identified. Hence, the keyhole was recorded clearly when the laser was off. Therefore, to better characterize the keyhole, we selected the first recorded image (the $0.7-\mathrm{ms}$ image) after the laser was turned off.

After manually identifying the top and bottom surfaces of the keyhole from the obtained camera image (see Figure 2(c)), the keyhole depth in one pulse was measured. The mass lost by ejection and vaporization caused a depth increment in each pulse. The depth increment, expressed as the difference in keyhole depth between two adjacent pulses, increased with increasing material-removal rate. Therefore, the depth increment is a characteristic feature of the material-removal rate.

Figure 3 shows the keyhole evolution during multi-pulse drilling. The effectiveness of the melt ejection reduced after the third pulse; consequently, the depth increments were larger during the first two pulses than during the remaining eight pulses, implying lower material-removal rate after the third pulse. Hence, the multi-pulse drilling process can be divided into two stages: a rapid penetration stage followed by a moderate penetration stage. During the rapid penetration stage (labeled A to C in Figure 3), the keyhole depth increased rapidly and was mainly driven by ejection. During the moderate penetration stage ( $\mathrm{C}$ to $\mathrm{E}$ in Figure 3 ), the keyhole depth increased at a more moderate rate. After the beginning of the third pulse, the keyhole depth increased linearly from time points $\mathrm{C}$ to D. During this linear growth period, the keyhole depth could be simplified as a linear function of pulse number. At a certain depth (marked by the arrow), the keyhole bulged due to multiple reflections on the keyhole wall and the erosive effect of the high temperature vapor. In the final phase of the evolution (time points $\mathrm{D}$ to $\mathrm{E}$ ), the keyhole depth was almost constant. The keyhole depths and depth increments of the pulses are listed in Table 1.

Figure 4 shows the keyhole evolution at different stages of its evolution during one pulse. The first pulse in the rapid penetration stage and the first pulse in the moderate penetration stage (the third pulse in the multi-pulse drilling) were selected for analysis.

The first pulse in the rapid penetration stage melted the material within the initial $0.2 \mathrm{~ms}$. Because there was no ejection, the material was mainly removed by vaporization and the keyhole was shallow [10]. When the ejection started, the keyhole evolved rapidly as shown in Figure 4(a). After the laser turn-off, melting and vaporization ceased but the molten material continued ejecting until the keyhole was nearly empty, forming a thin liquid layer as shown in Figure 4(b). At this time, the temperature rapidly decreased. The images in Figure 4(a) were recorded through both the bandpass filter and neutral filters, whereas those in Figure 4(b) were recorded through the neutral filter only.

The first pulse in the moderate penetration stage was unaccompanied by vapor or melt ejection over the hole entrance, but the keyhole evolved moderately as shown in Figure 4(c). When the laser was turned off, the molten material remained inside the keyhole, where it cooled naturally and slowly (see Figure 4(d)). The images in Figure 4(c) were recorded through both the band-pass filter and neutral filter, whereas those in Figure 4(d) were recorded through the neutral filter only. The laser power density was lower in this pulse than in the first pulse of the rapid penetration stage. Hence, the laser-induced light was weaker and further dimmed after filtering through both filters. Consequently, the keyhole was smaller at $0.6 \mathrm{~ms}$ than at $0.7 \mathrm{~ms}$.

As previously discussed, it was difficult to judge whether the highlighted region inside the keyhole (Figure 4(c)) was caused by vapor or the laser-induced light. To clarify this effect, the "sandwich" structure was replaced by a thick metal sheet (Figure 1(a)). When the thicker metal sheet was 


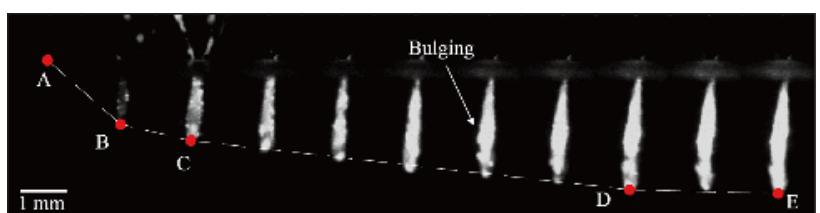

Figure 3 (Color online) Keyhole evolution during multi-pulse drilling.

Table 1 Keyhole depth and depth increment of each pulse during multipulse drilling

\begin{tabular}{ccc}
\hline Pulse number & Keyhole depth $(\mathrm{mm})$ & Depth increment $(\mathrm{mm})$ \\
\hline 1 & 1.38 & 1.38 \\
2 & 1.66 & 0.28 \\
3 & 1.92 & 0.26 \\
4 & 2.16 & 0.24 \\
5 & 2.34 & 0.17 \\
6 & 2.54 & 0.20 \\
7 & 2.66 & 0.11 \\
8 & 2.79 & 0.13 \\
9 & 2.86 & 0.07 \\
10 & 2.93 & 0.07 \\
\hline
\end{tabular}

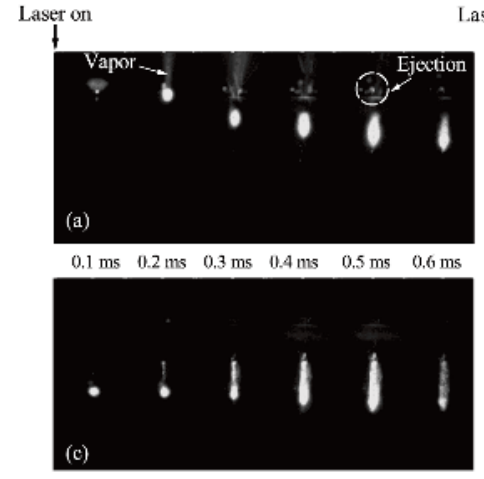

$\begin{array}{llllllll}0.1 & \mathrm{~ms} & 0.2 \mathrm{~ms} & 0.3 \mathrm{~ms} & 0.4 \mathrm{~ms} & 0.5 \mathrm{~ms} & 0.6 \mathrm{~ms} & 0\end{array}$

Figure 4 (Color online) Keyhole evolution during laser turn-on (a) and turn-off (b) in the first pulse, and laser turn-on (c) and laser turn-off (d) in the third pulse.

irradiated by the laser with unchanged parameters, the vaporization behavior inside the keyhole was clearly recorded as shown in Figure 5. The keyhole depths during the first four pulses are listed in Table 2. Comparing the keyhole depths in Tables 1 and 2, we find that the laser power density distribution exerted similar effects on the vaporization during multi-pulse drilling of the metal sheet and multi-pulse drilling of the "sandwich" structure.

From Figures 4(c) and 5(c), it can be deduced that the highlighted region inside the keyhole, observed from 0.3 to $0.5 \mathrm{~ms}$ in Figure 4(c), was caused by vapor. Therefore, the dominant material-removal mechanism changed from melt ejection to vaporization. At this time, the keyhole evolution during the pulses changed from fast to moderate. Hence, the
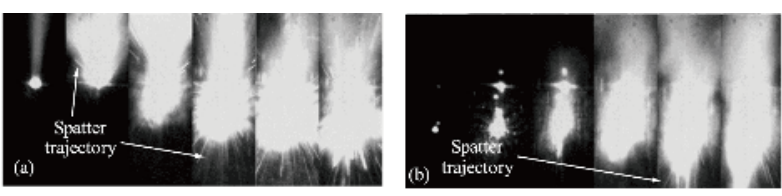

$\begin{array}{lllll}0.1 & \mathrm{~ms} & 0.2 \mathrm{~ms} \quad 0.3 \mathrm{~ms} \quad 0.4 \mathrm{~ms} & 0.5 \mathrm{~ms} \quad 0.6 \mathrm{~ms}\end{array}$

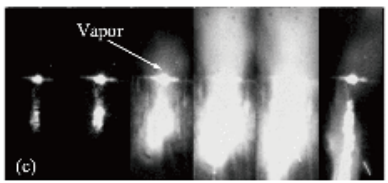

$\begin{array}{lllll}0.1 \mathrm{~ms} & 0.2 \mathrm{~ms} \quad 0.3 \mathrm{~ms} \quad 0.4 \mathrm{~ms} \quad 0.5 \mathrm{~ms} \quad 0.6 \mathrm{~ms}\end{array}$

$0.1 \mathrm{~ms} \quad 0.2 \mathrm{~ms} \quad 0.3 \mathrm{~ms} \quad 0.4 \mathrm{~ms} \quad 0.5 \mathrm{~ms} \quad 0.6 \mathrm{~ms}$

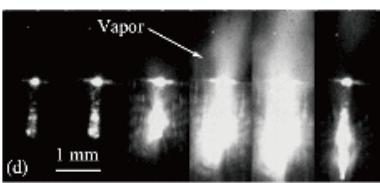

$\begin{array}{llllll}0.1 \mathrm{~ms} & 0.2 \mathrm{~ms} & 0.3 \mathrm{~ms} & 0.4 \mathrm{~ms} & 0.5 \mathrm{~ms} & 0.6 \mathrm{~ms}\end{array}$

Figure 5 Observation of the vaporization and ejection processes during the first pulse (a), second pulse (b), third pulse (c), and fourth pulse (d).

Table 2 Keyhole depths for first four pulses during multi-pulse drilling with metal sheet

\begin{tabular}{cc}
\hline Pulse number & Keyhole depth $(\mathrm{mm})$ \\
\hline 1 & 1.31 \\
2 & 1.58 \\
3 & 1.83 \\
4 & 2.05 \\
\hline
\end{tabular}

vaporized mass loss could be considered as the driving mechanism during the moderate penetration stage.

In a nutshell, the keyhole evolution in each pulse during the rapid penetration stage was driven by both ejection and vaporization, but was dominated by ejection. In the moderate penetration stage, the keyhole evolution was driven dominantly by vaporization.

\subsection{Effect of dimensionless laser power density on the driving mechanism}

The ejection and vaporization behavior depends on both the material properties and the laser power density of multipulse drilling [7]. Melt ejection is possible only when the laser power density exceeds a certain threshold value [27]. Increasing the laser power density enhances the vaporization-induced recoil pressure, improving the ejection efficiency and thinning the molten layer [28]. When the laser power density is below the threshold density, no ejection occurs and the transition from ejection-driven to vaporization-driven may be affected by the varying laser power density at the keyhole front.

The laser power density inside a Gaussian beam $I(r)$ can be calculated from the maximum laser power density in the center of the beam $I_{0}$. The equation is given by [29-31]

$I(r)=I_{0} \cdot \exp \left(\frac{-2 \cdot r^{2}}{(w / 2)^{2}}\right)$,

where $r$ denotes the radius from the beam center and $w$ is the beam diameter.

The maximum laser power density can be calculated from the peak power $P$ by eq. (2): 
$I_{0}=\eta \frac{2 \cdot P}{\pi \cdot(w / 2)^{2}}$

where $\eta$ means the absorption coefficient. In eqs. (1) and (2), the beam diameter $w$ can be calculated from the vertical distance to the focus position $z$ by eq. (3):

$w(z)=w_{0} \cdot \sqrt{1+\left(\frac{z}{z_{0}}\right)^{2}}$,

where $w_{0}$ and $z_{0}$ are the focus diameter and the Rayleigh length, respectively.

To investigate how the material properties and laser power density influence the ejection and vaporization behavior, we computed the dimensionless laser power density as [32]:

$I^{\prime}=\frac{0.5 \cdot w(z) \cdot I_{0} \cdot C_{p}}{h_{\mathrm{lv}} \cdot k}$,

where the dimensionless power density $I^{\prime}$ defines the ratio of the enthalpy increment caused by absorbing the laser energy to the latent heat of vaporization. $C_{p}, h_{\mathrm{lv}}$, and $k$ denote the specific heat, latent heat of vaporization, and thermal conductivity of the material, respectively.

In eq. (4), we assumed the keyhole depths listed in Table 1 and the related thermophysical parameters listed in Table 3. The dimensionless laser power densities at the beginning of the ten pulses in the multi-pulse drilling are listed in Table 4.

To further investigate the effect of the dimensionless laser power density on the driving mechanism, we performed single-pulse laser drilling experiments using the first four dimensionless laser power densities in Table 4 with a defocus distance of 0 . The observed vaporization and ejection behaviors are shown in Figure 6. The vapor and ejection were

Table 3 Thermophysical parameters of 304 stainless steel [33]

\begin{tabular}{cc}
\hline Thermophysical parameter & Value \\
\hline Liquid density $\left(\mathrm{kg} / \mathrm{m}^{3}\right)$ & $7.2 \times 10^{3}$ \\
Absorption coefficient & 0.27 \\
Viscosity $(\mathrm{kg} /(\mathrm{m} \mathrm{s}))$ & 0.1 \\
Solidus temperature $(\mathrm{K})$ & 1697 \\
Melting temperature $(\mathrm{K})$ & 1727 \\
Boiling temperature $(\mathrm{K})$ & 3200 \\
Enthalpy of solid at melting point $(\mathrm{J} / \mathrm{kg})$ & $1.20 \times 10^{6}$ \\
Enthalpy of liquid at melting point $(\mathrm{J} / \mathrm{kg})$ & $1.26 \times 10^{6}$ \\
Latent heat of vaporization $(\mathrm{J} / \mathrm{kg})$ & $6.34 \times 10^{6}$ \\
Solid specific heat $(\mathrm{J} /(\mathrm{kg} \mathrm{K}))$ & 711.8 \\
Liquid specific heat $(\mathrm{J} /(\mathrm{kg} \mathrm{K}))$ & 837.4 \\
Thermal conductivity of solid $(\mathrm{J} /(\mathrm{m} \mathrm{s} \mathrm{K}))$ & 19.26 \\
Effective thermal conductivity of liquid $(\mathrm{J} /(\mathrm{m} \mathrm{s} \mathrm{K}))$ & 209.3 \\
Convective heat transfer coefficient $\left(\mathrm{W} /\left(\mathrm{m}^{2} \mathrm{~K}\right)\right)$ & 40 \\
Temperature coefficient of surface tension $(\mathrm{N} /(\mathrm{m} \mathrm{K}))$ & $-0.43 \times 10^{-3}$ \\
Coefficient of thermal expansion & $1.96 \times 10^{-5}$ \\
Surface tension coefficient $(\mathrm{N} / \mathrm{m})$ & 1.872 \\
\hline
\end{tabular}

Table 4 Dimensionless laser power densities at the beginning of each pulse in the multi-pulse drilling

\begin{tabular}{cc}
\hline Pulse number & Dimensionless laser power density \\
\hline 1 & 16.39 \\
2 & 12.04 \\
3 & 10.96 \\
4 & 10.08 \\
5 & 9.33 \\
6 & 8.84 \\
7 & 8.31 \\
8 & 8.04 \\
9 & 7.76 \\
10 & 7.61 \\
\hline
\end{tabular}
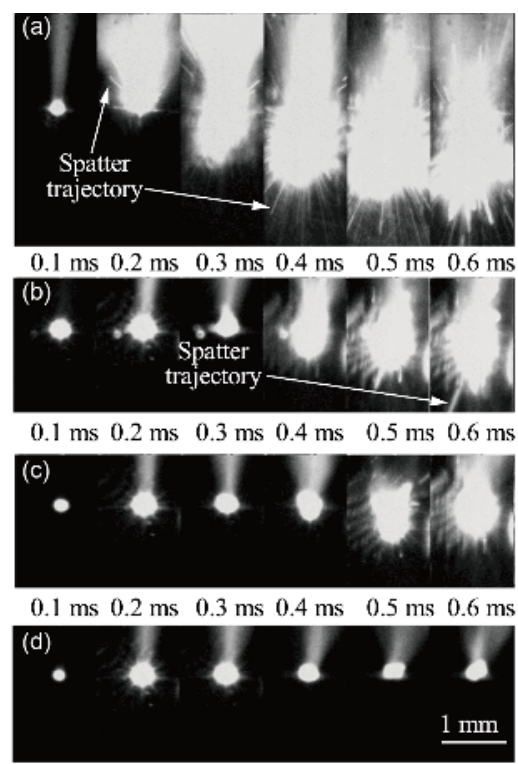

$0.1 \mathrm{~ms} \quad 0.2 \mathrm{~ms} \quad 0.3 \mathrm{~ms} \quad 0.4 \mathrm{~ms} \quad 0.5 \mathrm{~ms} \quad 0.6 \mathrm{~ms}$

Figure 6 Keyhole evolution in single-pulse laser drilling with different dimensionless laser densities. (a) 16.39, (b) 12.04, (c) 10.96, and (d) 10.08 .

intense at dimensionless laser power densities above 12.04. Below 12.04, only metal vapor was observed. The effects of the dimensionless laser power density on the driving mechanism were similar in the single-pulse and multi-pulse drilling processes (c.f. Figures 5 and 6).

To better understand the effect of the dimensionless laser power density on the driving mechanism, a numerical simulation was performed under the following assumptions [34]: (1) The laser power density was spatially Gaussian. (2) The shielding effect of the laser-induced plasma, multiple reflections, and divergence of the laser beam could be ignored. (3) The dynamic behavior of the melt flow and mass loss due to ejection were ignored to simplify the calculation. The mass loss was considered to be dominated by vaporization.

The speed of the gas-liquid interface was defined as [35]: 
$F_{e}=M_{\text {vap }} / \rho_{1}$,

where $\rho_{1}$ is the density of the material in liquid state. The term on the left-hand side is the net mass loss due to vaporization [36], which can be calculated as [35]:

$M_{\text {vap }}=\rho_{\mathrm{s}} \sqrt{R T_{\mathrm{s}} / 2 \pi}-\rho_{\mathrm{v}} \sqrt{R T_{\mathrm{v}} / 2 \pi} \beta F(m)$,

where $\rho_{\mathrm{s}}$ and $\rho_{\mathrm{v}}$ are the densities of the material in the solid and vapor states, respectively, $T_{\mathrm{s}}$ is the surface temperature at the gas-liquid interface, $T_{\mathrm{v}}$ is the vapor temperature near the Kundsen layer, $R$ is the gas constant, and $\beta$ and $F(m)$ are respectively defined as follows:

$\beta=\frac{2\left(2 m^{2}+1\right) \sqrt{T_{\mathrm{v}} / T_{\mathrm{s}}}-2 \sqrt{\pi} m}{F(m)+\sqrt{T_{\mathrm{v}} / T_{\mathrm{s}}} G(m)}$,

$F(m)=\sqrt{\pi} m(-1+\operatorname{erf}(m))+\exp \left(-m^{2}\right)$,

with $G(m)$ defined as:

$G(m)=\left(2 m^{2}+1\right)(1-\operatorname{erf}(m))-\frac{2 m}{\sqrt{\pi}} \exp \left(-m^{2}\right)$,

and $m$ defined as:

$m=\sqrt{\gamma_{\mathrm{v}} / 2} M_{\mathrm{v}}$.

where $\gamma_{\mathrm{v}}$ is the specific heat ratio and $M_{\mathrm{v}}$ is the Mach number.

The surface was tracked by the level-set method [37]. The level-set function is given by

$\frac{\partial \phi}{\partial t}+F_{e}|\Delta \phi|=0$

where $\phi$ is the interface function, representing the gas-liquid interface when $\phi$ is 0 .

The conservation equations of mass, momentum, and heat are given by eqs. (12)-(14), respectively:

$\frac{\partial \rho}{\partial t}+\frac{\partial\left(\rho u_{i}\right)}{\partial x_{i}}=0$

$\frac{\partial\left(\rho u_{i}\right)}{\partial t}+\frac{\partial\left(\rho u_{i} u_{j}\right)}{\partial x_{j}}=\frac{\partial}{\partial x_{i}}\left(\mu \frac{\partial u_{j}}{\partial x_{i}}\right)+q_{s 1}$,

$q_{s 1}=-\frac{\partial p}{\partial x_{i}}+\frac{\partial}{\partial x j}\left(\mu \frac{\partial u_{j}}{\partial x_{i}}\right)-K_{0} \frac{\left(1-f_{l}\right)^{2}}{f_{l}^{3}+B} u_{i}$,

$\frac{\partial\left(\rho C_{p} T\right)}{\partial t}+\frac{\partial\left(\rho u_{i} C_{p} T\right)}{\partial x_{i}}=\frac{\partial}{\partial x_{i}}\left(k \frac{\partial T}{\partial x_{i}}\right)-q_{s 2} \delta(\phi)$,

$q_{s 2}=q_{\mathrm{laser}}-\rho_{l} h_{\mathrm{lv}} F_{e}-h_{c}\left(T-T_{\infty}\right)-\sigma \varepsilon\left(T^{4}-T_{\infty}^{4}\right)$,

where $\rho$ is the density of the material, $u$ is the velocity of the molten material, $p$ is the pressure, $q_{\text {laser }}$ represents the input laser power density, $h_{\mathrm{c}}$ is the convective heat-transfer coefficient, and $\sigma$ and $\varepsilon$ denote the Stefan-Boltzmann constant and emissivity, respectively [38]. $T_{\infty}$ represents the atmospheric temperature. The third term on the right-hand side of eq. (13b) describes the frictional dissipation in the mushy region [39]. Meanwhile, the second term on the right-hand side of eq. (14a) represents the actual laser energy on the gasliquid interface, and the first and second terms in eq. (14b) are the laser input energy and the energy lost by vaporization, respectively. The remaining terms in eq. (14b) represent the energy losses by convection and radiation, respectively. The delta function $\delta(\phi)$ of the level-set values includes the boundary conditions of the gas-liquid interface and is defined as:

$\delta(\phi)= \begin{cases}1, & \phi=0 \\ 0, & \phi \neq 0\end{cases}$

The related thermophysical parameters are listed in Table 3, and the simulated and experimental depth increments of the keyhole induced by a laser pulse are presented in Figure 7. At dimensionless power densities above 12.04, the simulated and experimental depth increments were very different, because the simulation ignored the effect of ejection on the keyhole evolution. When the dimensionless laser power density was 16.39 , the experimental depth increment was $1.38 \mathrm{~mm}$, and the simulated depth increment driven by vaporization was $0.38 \mathrm{~mm}$ (27.5\% of the experimental value). In other words, approximately $72.5 \%$ of the depth increment was driven by ejection. When the dimensionless laser power density was 12.04, the experimental and simulated depth increments were 0.33 and $0.24 \mathrm{~mm}$, respectively, meaning that approximately $27.3 \%$ of the depth increment was driven by ejection. When the dimensionless laser power density was below 12.04, the simulated depth increments were close to the experimental depth increments, and the keyhole evolution throughout the pulse was dominantly driven by vaporization.

The above analysis suggests different driving mechanisms in multi-pulse and single-pulse laser drilling. In single-pulse drilling, the keyhole evolution appears to be dominated by ejection [40], with temporary dominance of vaporization in the mild melting stage or the hole expansion stage [10]. In multi-pulse irradiation, the keyhole evolution and its driving

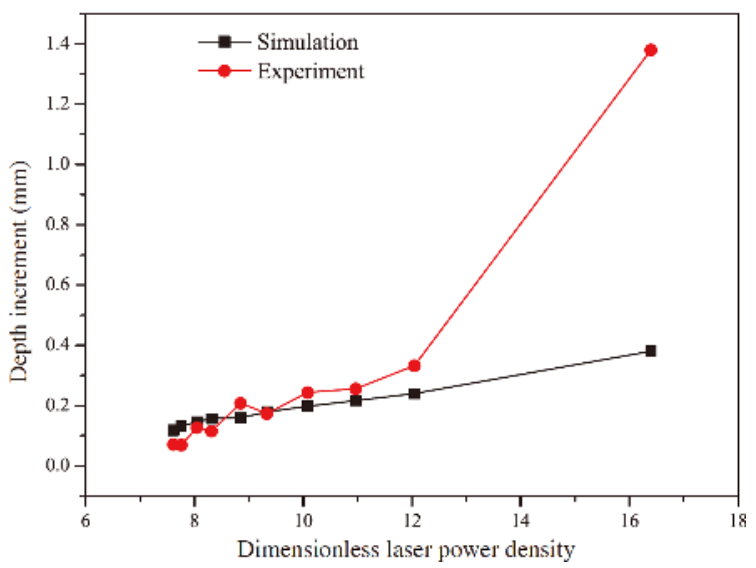

Figure 7 (Color online) Effect of dimensionless laser power density on the depth increment of the keyhole during one pulse of multi-pulse drilling. 
mechanism during the rapid penetration stage were similar to those in single-pulse drilling. However, during a pulse in the moderate penetration stage of multi-pulse irradiation, the keyhole evolution was driven by vaporization.

\section{Conclusion}

In summary, the keyhole evolution during multi-pulse drilling was observed in a time-resolved system with a "sandwich" structure. The evolutionary characteristics suggested a two-stage evolution progressing from rapid penetration to moderate penetration. After observing the ejection and vaporization behavior in a drilled metal sheet, the keyhole evolution was found to be dominated by ejection during the rapid penetration stage, and by vaporization during the moderate penetration stage. The driving mechanism was affected by the dimensionless laser power density, as confirmed in a single-pulse experiment and numerical simulation. Ejection dominated at dimensionless laser power densities above 12.04, causing rapid evolution of the keyhole. In contrast, vaporization dominated at dimensionless laser power densities below 12.04, leading to a linear growth and a slow evolution. This work has revealed the keyhole evolution and its driving mechanism, providing new insights into the physical processes during the multi-pulse laser drilling process.

This work was supported by the National Natural Science Foundation of China (Grant Nos. 11672304, 11502269, 11272316, and 11272317), and the Plan of Beijing Municipal Commission of Science and Technology (Grant No. Z181100003818015).

1 G. D. Gautam, and A. K. Pandey, Opt. Laser Tech. 100, 183 (2018).

2 J. Zhu, H. L. Zhan, K. Zhao, X. Y. Miao, Q. Zhou, and W. Z. Yue, Sci. China-Phys. Mech. Astron. 62, 974222 (2019).

3 J. Tu, A. G. Paleocrassas, N. Reeves, and N. Rajule, Opt. Lasers Eng. 55, 275 (2014).

4 S. Döring, J. Szilagyi, S. Richter, F. Zimmermann, M. Richardson, A Tünnermann, and S. Nolte, Opt. Express 20, 27147 (2012).

5 Y. Yan, L. Ji, Y. Bao, and Y. Jiang, J. Mater. Proc. Tech. 212, 1257 (2012).

6 W. Perrie, A. Rushton, M. Gill, P. Fox, and W. O’Neill, Appl. Surf. Sci. 248, 213 (2005).

7 K. T. Voisey, S. S. Kudesia, W. S. O. Rodden, D. P. Hand, J. D. C.
Jones, and T. W. Clyne, Mater. Sci. Eng.-A 356, 414 (2003).

8 R. K. Ganesh, A. Faghri, and Y. Hahn, Int. J. Heat Mass Transf. 40, 3351 (1997).

9 R. K. Ganesh, A. Faghri, and Y. Hahn, Int. J. Heat Mass Transf. 40, 3361 (1997).

10 M. Chen, Y. Wang, G. Yu, D. Lan, and Z. Zheng, Appl. Phys. Lett. 103, 194102 (2013)

11 H. Ki, P. S. Mohanty, and J. Mazumder, J. Phys. D-Appl. Phys. 34, 364 (2001).

12 H. Ki, J. Appl. Phys. 107, 104908 (2010).

13 B. S. Yilbas, and M. Sami, J. Phys. D-Appl. Phys. 30, 1996 (1997).

14 F. Q. Yang, Sci. China-Phys. Mech. Astron. 60, 104621 (2017).

15 Y. Zhang, S. Li, G. Chen, and J. Mazumder, Opt. Laser Tech. 48, 405 (2013).

16 V. V. Semak, G. A. Knorovsky, D. O. MacCallum, and R. A. Roach, J. Phys. D-Appl. Phys. 39, 590 (2006).

17 M. Brajdic, M. Hermans, A. Horn, and I. Kelbassa, Meas. Sci. Technol. 19, 105703 (2008).

18 P. Gu, S. B. Liu, S. Liu, and H. Y. Song, Appl. Surf. Sci. 293, 80 (2010).

19 A. F. H. Kaplan, Appl. Phys. Lett. 101, 151605 (2012).

20 T. Z. Zhang, Z.-C. Jia, H. C. Cui, D. H. Zhu, X. W. Ni, and J. Lu, Chin. Phys. B 25, 054206 (2016).

21 G. K. L. Ng, and L. Li, Opt. Laser Tech. 33, 393 (2001).

22 K. Salonitis, A. Stournaras, G. Tsoukantas, P. Stavropoulos, and G. Chryssolouris, J. Mater. Proc. Tech. 183, 96 (2007).

23 S. Sharma, V. Mandal, S. A. Ramakrishna, and J. Ramkumar, J. Mater. Proc. Tech. 262, 131 (2018)

24 N. D. Pandey, H. S. Shan, and A. Bharti, Int. J. Adv. Manuf. Technol. 28, 863 (2006)

25 K. Walther, M. Brajdic, and E. W. Kreutz, Int. J. Adv. Manuf. Technol. 35, 895 (2006)

26 M. Zhang, G. Chen, Y. Zhou, and S. Li, Opt. Express 21, 19997 (2013).

27 Y. N. Zang, X. W. Ni, and B. Han, J. Appl. Phys. 116, 013104 (2014).

28 A. Luft, U. Franz, L. Emsermann, and J. Kaspar, Appl. Phys. A 63, 93 (1996).

29 B. Adelmann, and R. Hellmann, J. Mater. Proc. Tech. 221, 80 (2015).

30 S. N. Pan, G. Yu, X. L. He, S. X. Li, and R. Chen, Sci. China-Phys. Mech. Astron. 61, 104621 (2018).

31 Y. J. Zhang, G. Yu, and X. L. He, Sci. China-Phys. Mech. Astron. 55, 1431 (2012).

32 Y. Yang, Z. Chen, and Y. Zhang, Int. J. Therm. Sci. 107, 141 (2016)

33 X. He, P. W. Fuerschbach, and T. D. Roy, J. Phys. D-Appl. Phys. 36, 1388 (2003)

34 A. Kar, and J. Mazumder, J. Appl. Phys. 68, 3884 (1990).

35 T. Ytrehus, and S. Østmo, Int. J. Multiph. Flow 22, 133 (1996).

36 X. He, T. D. Roy, and P. W. Fuerschbach, J. Phys. D-Appl. Phys. 36, 3079 (2003)

37 S. Osher, and J. A. Sethian, J. Comput. Phys. 79, 12 (1988).

38 H. Liu, X. L. He, G. Yu, Z. B. Wang, S. X. Li, C. Y. Zheng, and W. J. Ning, Sci. China-Phys. Mech. Astron. 58, 104701 (2015).

39 S. Asai, and I. Muchi, Trans. Iron Steel Inst. Jpn. 18, 90 (1978).

40 R. E. Wagner, J. Appl. Phys. 45, 4631 (1974). 